\title{
A Two-Temperature Model for LBVs
}

\author{
J. H. Guo ${ }^{1,2}, \mathbf{Y} \cdot \mathbf{L i}^{1}$, and H. G. Shan ${ }^{1}$ \\ ${ }^{1}$ National Astronomical Observatories/Yunnan Observatory, Chinese Academy of Sciences, \\ P.O. Box 110, Kunming 650011, China \\ email: guojh@ynao.ac.cn \\ ${ }^{2}$ Graduate School of Chinese Academy of Sciences
}

\begin{abstract}
The continuum energy distributions of R127 and R110 in the outburst phase are fitted by use of a optically envelope model. Both stars show two peaks in the continuum energy distributions in which one lies in the short-wavelength range (near $1250 \AA$ ) and the other in the optical band. We suggest that the fluxes in the UV and optical bands may have different origins: the UV flux comes from the central star and the optical flux comes from the expanded optically envelope. We construct such a model for LBVs with the use of two LTE atmosphere models with different temperatures, and find it to be in satisfactory agreement with the observed spectral energy distributions of R127 and R110.
\end{abstract}

Keywords. stars: mass loss, variables: other

\section{Introduction}

Luminous blue variables (LBVs) are a separate class of massive stars. They are very evolved post main sequence stars or post red super giants. Their most distinct character is irregular variations of brightness on different timescales. The amplitudes of such variations vary from very small to a few magnitudes, when the timescales vary from the order of days to a few hundred years (van Genderen 2001). A general discussion of the LBV phenomenon can be found in Nota \& Lamers (1997). Recent investigations focus on the instability of the moderate variations, which is characterized by visual magnitude changes of 1 to $2 \mathrm{mag}$ and timescales from years to decades. Whereas the normal LBVs at the quiescent phase occupy a wide temperature range, the eruptive LBVs are almost in a vertical strip on the HR diagram (Wolf 1989). Their continuum radiation shows the spectra of hot super giants at the minimum or quiescent phase. During the visual maximum period, the atmosphere of an LBV resembles a much cooler supergiant of spectral type A or F (Humphreys \& Davidson 1994).

Investigations on the continuum spectra of the LBVs are rare but desirable, because they may indicate the basic configuration and physical conditions of the LBVs. Stahl et al. (1983, 1990), Shore et al. (1996), and Szeifert et al. (1993) have studied R127, R110, R40, and AG Car. Their results hint a general feature, namely the brightness variations in the UV and optical bands are anti-correlated. However, an important feature on the continuum spectra of R127 and R110, namely the over-luminous component in the short-

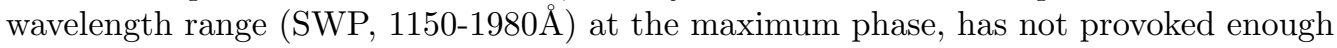
interest of investigators up to now. Figure 1 shows the continuum energy distributions of R127 and R110 during the maximum phase. Evidently, the continuum show two peaks. On the other hand, the non-spherically symmetric wind(mass loss) of some LBVs have been identified by many astronomers(Nota et al., 1992; Leitherer et al., 1994; SchulteLadbeck et al., 1993). It is usually assumed that the deposition of the matter can form optically thick regions (Davidson 1987), but optically thin regions can also be formed simultaneously due to the anisotropy of the stellar wind or mass ejection. This shows 
Table 1. The photometric data of R127

\begin{tabular}{|c|c|c|c|c|c|c|c|c|c|c|}
\hline Data & System & U & B & V & $\mathrm{J}$ & $\mathrm{H}$ & $\mathrm{K}$ & $\mathrm{L}$ & State & Ref. \\
\hline Dec. 1969 & BVRI & & & 11.16 & & & & & $\min$ & Mendoza (1970) \\
\hline Feb. 16, 1983 & UBV & $\begin{array}{c}9.21 \\
\mathrm{u}\end{array}$ & $\begin{array}{c}10.23 \\
\mathrm{v}\end{array}$ & $\begin{array}{c}10.13 \\
\mathrm{~b}\end{array}$ & $\begin{array}{c}9.76 \\
\mathrm{y}\end{array}$ & 9.58 & 9.4 & 8.850 & $\min *$ & Stahl et al. (1983) \\
\hline Nov. 30, 1988 & uvby & 9.82 & 9.36 & 9.10 & 8.84 & & & & $\max$ & LTPV \\
\hline
\end{tabular}

a relative minimum during the interval of 1983-1992

Table 2. The photometric data of R110

\begin{tabular}{clllllllllll}
\hline \hline Data & System & U & B & V & R & I & J & H & K & State & Ref. \\
Jan. 1989 & UBV & 10.26 & 10.34 & 9.99 & 9.77 & 9.53 & 9.28 & 9.19 & 9.07 & max & $\begin{array}{l}\text { Stahl } \text { et al. } \\
\text { (1990) }\end{array}$ \\
& & & & & & & & & & & \\
Sep. 26, 1990 & uvby & u & v & b & y & & & & & & \\
\hline
\end{tabular}

that the asymmetric mass loss can result in a coexistence of optically thin and thick regions around the star, which allows the radiation from the star to be seen by observers. We suggest, that the over-luminous continuum in the SWP range could be a part of the radiation from the central star. The radiation from the optically thick regions induced by the asymmetric mass loss concentrates on the optical band, because these regions are far from the central star and therefore have lower temperatures. The combination of both radiation components may reproduce the observed continuum energy distributions. So, we decided to fit the observed continuum spectra of the LBVs during the maximum phase with a model of two stellar atmospheres, in which one denotes the central star and the other represents the optically thick regions formed around the central star by the anisotropic mass loss.

\section{Observations}

The photometry of R127 (=HDE 269858) with the UBV photometer has already been published and summarized by Stahl et al. (1983) and Spoon et al. (1994). In addition, a long-term project to monitor slow variations (LTPV) originated by Sterken (1983) also included R127 and R110, which was done in the Strömgren uvby-system. In Table 1 we summarize these observational data for R127. The photometric history of R110 is summarized in Table 2 .

All spectra discussed in this paper have been obtained through the database of the

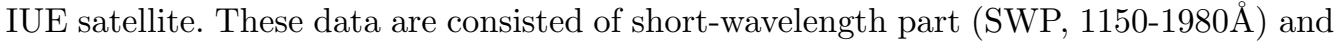
long-wavelength part (LWP and LWR, 1850-3350 $)$ in low $(\mathrm{R}=300)$ and high $(\mathrm{R}=10$ 000) resolution modes. All raw data have been reduced by the NEW SPECTRAL IMAGE PROCESSING SYSTEM (ref. IUE NEWSIPS manual; Nichols \& Linsky 1996). In Table 3 we collect the observe time, data format, exposure time, and so on.

\section{Continuum energy distributions}

The ground based photometric data combined with the IUE data were used to constitute the continuum energy distributions. We corrected the observed IUE spectra for the interstellar reddening using the interstellar law of Savage and Mathis (1979) and Nandy et al. (1981) for the galactic foreground extinction and the absorption within the LMC. Considering both stars in the LMC, the same value of the interstellar extinction 
Table 3. IUE observations of R127 and R110.

\begin{tabular}{lcccccccc}
\hline Object & Date & Camera & Aperture $^{a}$ & Dispersion $^{b}$ & $\begin{array}{c}\text { Exposure } \\
\text { time[min] }\end{array}$ & $\begin{array}{c}\text { Image } \\
\text { number }\end{array}$ & State \\
\hline R127 & 1989 Feb.11 & LWP & L & L & 3 & 15013 & max \\
& 1989 Feb.11 & SWP & L & L & 15 & 35533 & max \\
& 1983 Mar.02 & SWP & L & L & 10 & 19372 & min \\
& 1983 Mar.02 & LWR & L & L & 6 & 15407 & min \\
R110 & 1989 Apr.25 & LWP & L & L & 30 & 15404 & $\max$ \\
& 1989 Apr.25 & SWP & L & L & 75 & 36088 & $\max$ \\
\hline
\end{tabular}

${ }^{a}$ Aperture: $\mathrm{L}=$ Large $^{b}$ Dispersion: $\mathrm{L}=$ Low

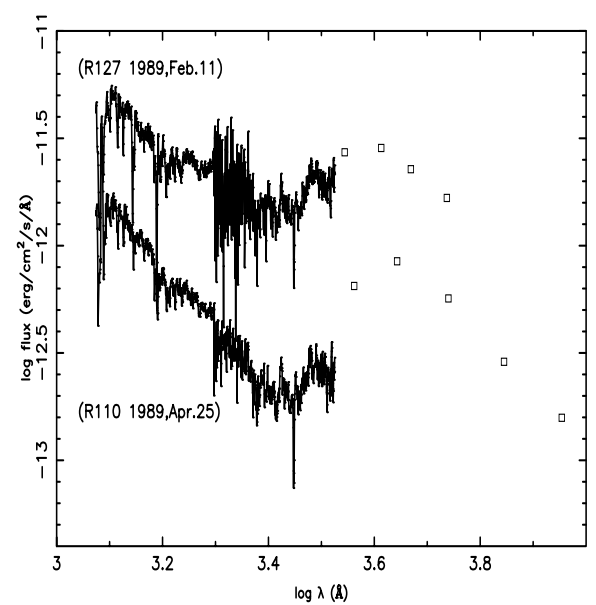

Figure 1. The de-reddened continuum energy distributions of R127 and R110 in the eruptive phase.

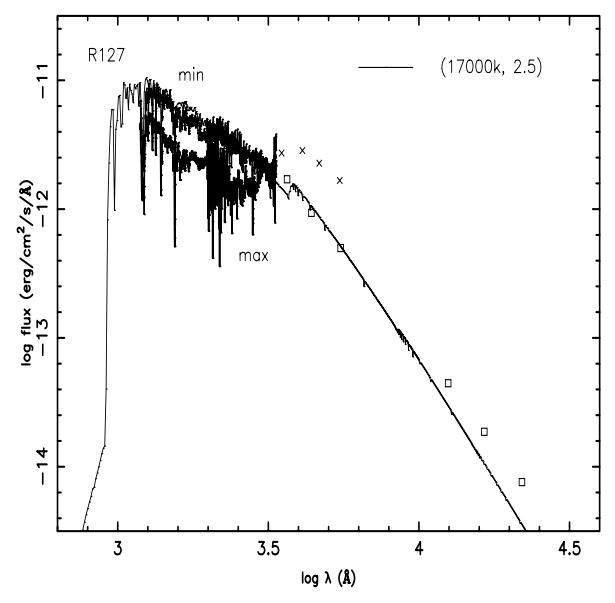

Figure 2. The spectral energy distributions of R127 at the minimum and maximum states. The crosses denote the photometric data at the maximum state, and the diamonds are at the minimum state.

was used. The standard value $\mathrm{E}(\mathrm{B}-\mathrm{V})=0.05$ was used for the galactic foreground extinction, and the value $\mathrm{E}(\mathrm{B}-\mathrm{V})=0.10$ was used for the LMC. The photometric data were de-reddened using the reddening law of Code et al. (1976) and Leitherer and Wolf (1984) for the visual and infrared bands, respectively. For the Strömgren uvby measurements the absolute calibration was obtained from Gray (1998).

\section{1. $R 127$}

A minimum state spectral energy distribution of R127 on March 2, 1983 is shown in Fig. 2. We fitted the continuum energy distribution with an LTE atmosphere model of $T_{\text {eff }}=17000 \mathrm{~K}, R=135 R_{\odot}$, and $\log g=2.5$, which is almost identical with the result of Stahl et al. (1983). Subsequently, R127 entered a period of outburst till 1989 during which the visual brightness increased slowly with the decrease of the UV flux. The continuum energy distributions of the two states (cf. Fig. 2) are similar in the short wavelength part, which could reflect the correlation between the quiescent and outburst states. Evidently, any atmosphere model cannot fit the essential profile of the continuum in the outburst phase due to the existence of two peaks. The first peak, which is located at about $1250 \AA$, reflects the feature of a B-type star ( R127 itself). The second peak emerges around $\mathrm{u}$ band in the Strömgren system as a normal A-type star (the optically thick regions in the wind). We have already known that the flux in the SWP at the 


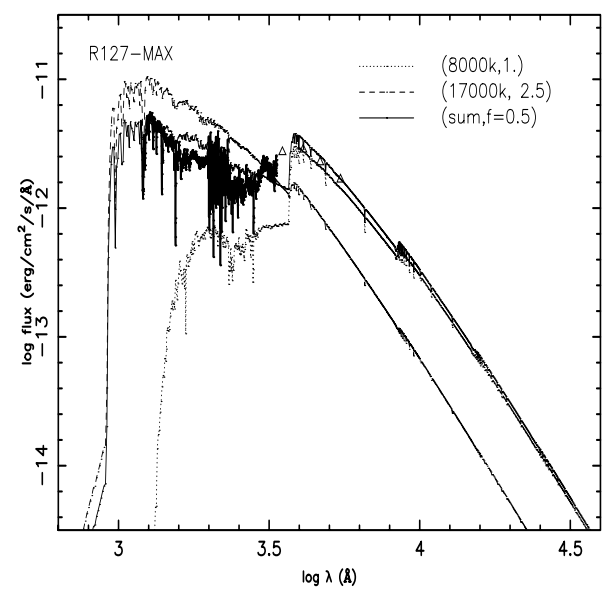

Figure 3. The model of the circumstellar envelope for R127 at the outburst phase. The continuum energy distribution is fitted by two atmosphere models of $T_{\text {eff }}=8000$ $\mathrm{K}$ (dotted line) and $T_{\text {eff }}=17000 \mathrm{~K}$ (dashedline), and $f=0.5$. The absolute fluxes at the effective wavelengths of the Strömgren uvby filters are given by triangles.

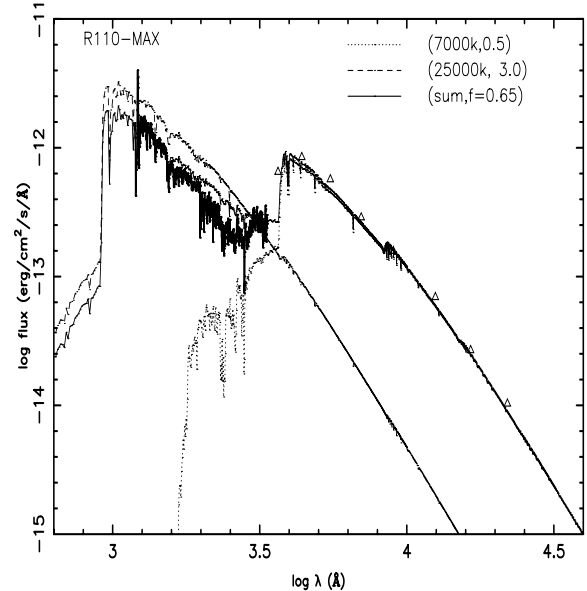

Figure 4. The model of R110 at the maximum phase. The continuum energy distribution is fitted by two atmosphere models of $T_{\text {eff }}=7000 \mathrm{~K}$ (dotted line) and $T_{\text {eff }}=25000 \mathrm{~K}$ (dashed line), and $f=0.65$.

minimum state is higher than that at the maximum state, thus the overall continuum energy distribution at the maximum state can be constructed with a permeating factor f, which measures how much light from the central star can permeate the optically thin regions in the wind and finally arrive at observers. Simply, the model in the quiescent state will be multiplied by the factor $\mathrm{f}$ (less than 1) to account for the observed SWP continuum in the outburst phase. Finally, the model at the outburst phase is constructed by adding the model of an A-type star to the modified minimum model of the central star (multiplied by factor $\mathrm{f}$ ). We fitted the profile of the optical spectrum at the outburst phase with a model of $T_{\text {eff }}=8000 \mathrm{~K}, R=485 R_{\odot}$ and $\log g=1$, which represented the average physical parameters of the optically thick regions. In our numerical experiments, we found that the permeating factor $f=0.5$ was appropriate to recover most characters of the observations of R127. The fitted result is shown in Fig. 3, which shows satisfactory agreement with the observations.

\section{2. $R 110$}

R110 increased its visual brightness by $0.5 \mathrm{mag}$ from 1980 to 1989 (Stahl et al. 1990). However, the physical parameters in the minimum phase are not known clearly due to the rare observations. Around 1989 R110 attained the maximum phase during which the continuum energy distribution appeared like R127 (cf. Fig. 4). Therefore, we fitted its continuum spectrum with the same technique for R127. Over the visual spectral rang a model of $T_{\text {eff }}=7000 \mathrm{~K}, R=350 R_{\odot}$ and $\log g=0.5$ was applied, which was comparable with the parameters derived by Stahl et al. (1990). For the whole continuum including the UV and visual bands the second component of $T_{\text {eff }}=25000 \mathrm{~K}, R=27 R_{\odot}$, and $\log$ $g=3.0$ was adopted, and the permeating factor $\mathrm{f}$ was chosen to be 0.65 . The result is shown in Fig. 4, which is satisfactory consistent with the observations. 


\section{Conclusions}

In this paper we discuss the continuum energy distributions of R127 and R110. We point out that the over-luminous continuum in the SWP range is the contribution from the central star itself due to the asymmetry of the external matter of the star. The light emitted from the central star leaks out through the optically thin regions of the nonhomogeneous envelope. Based on this detailed research we conclude that the continuum spectra of both stars at the outburst phase are composed of the radiation fluxes of the central star and the optically thick matter around the star. They make up the observed UV and optical continuum spectra, respectively.

\section{Acknowledgements}

We are grateful to P.S. Chen and S.H. Gu for the data process, and T. Szeifert for kind advice to transform the uvby magnitudes into absolute fluxes.

\section{References}

Code, A.D., Davis, J., Bless, R.C., \& Brown., R.H. 1976, ApJ 203, 417

Davidson, K. 1987, ApJ 317, 760

van Genderen, A.M. 2001, A\&\&A 366, 508

Gray, R.O. 1998, $A J$ 116, 482

Humphreys, R.M. \& Davidson, K. 1994, PASP 106, 704

Leitherer, C. \& Wolf, B. 1984, A\&A 132, 151

Leitherer, C., Allen, R., Altner, B., Damineli, A., Drissen, L., Idiart, T., Lupie, O., Nota, A., Robert, C., Schmutz, W., \& Shore, S.N. 1994, ApJ 428, 292

Nandy, K., Morgan, D.H., Willis, A.J., Wilson, R., \& Gondhalekar, P.M. 1981, MNRAS, 196, 955

Nichols, J.S. \& Linsky, J.L. 1996, AJ 111, 517

Nota, A., \& Lamers, H.J.G.L.M., eds. 1997, Luminous Blue Variables: Massive Stars in Transition, ASP Conference Series, 120

Nota, A., Leitherer, C., Clampin, M., Greenfield, P., \& Golinowski, D.A. 1992, ApJ 398, 621

Savage, B.D. \& Mathis, J.S. 1979, Ann. Rev. Astron. Astrophys. 17, 73

Schulte-Ladbeck, R.E., Leitherer, C., Clayton, G.C., Robert, C., Meade, M.R., Drissen, L., Nota, A., \& Schmutz, W. 1993, ApJ 407, 723

Shore, S.N., Altner, B., \& Waxin, I. 1996, AJ 112, 2744

Spoon, H.W.W., de Koter, A., Sterken, C., Lamers, H.J.G.L.M., \& Stahl, O. 1994, A\&AS 106, 141

Stahl, O., Wolf, B., Klare, G., Cassatella, A., Krautter, J., Persi, P., \& Ferrari-Toniolo, M. 1983, A\&SA 127, 49

Stahl, O., Wolf, B., Klare, G., Jüttner, A., \& Cassatella, A. 1990, A\&\&A 228, 379

Sterken, C. 1983, ESO The Messenger, No. 33, 10

Szeifert, T., Stahl, O., Wolf, B., Zickgraf, F.J., Bouchet, P., \& Klare, G. 1993, A $\& A$ 280, 508

Wolf, B. 1989, A\&GA 217, 87 